\title{
Performances of Micro-Endoscopy in Less Invasive Surgery
}

\author{
Depeursinge $\mathrm{Ch}$., Conde $\mathrm{R}$.
}

Institut d'Optique Appliquée, EPFL, CH-1015 Lausanne, Switzerland.

\section{ABSTRACT:}

Microendoscopy (diameter of the endoscope possibly less than $0.5 \mathrm{~mm}$ ) meets a number of needs in medical research and practise, which are not fulfilled yet by conventional endoscopy and opens a bright field of investigations and treatments in less invasive surgery. The microendoscope image quality, however, must still be improved. It suffers from several limitations imposed by the physical laws governing the propagation of light in the fiber guide. It is also degraded by the input lens and its interface with the fiber. MTF measurements have been performed to analyse the performances of each component of the microendoscope.

\section{INTRODUCTION:}

Microendoscopy is a class of endoscopes with diameters less than 1 . $\mathrm{mm}$ (possibly up to less than $0.5 \mathrm{~mm}$ ). A typical realization is shown on fig. 1 . It aims to investigate and treat, without major injury, almost every organs and tissues in the human body. Medical applications of microendoscopy are therefore important and numerous, in particular in view of the rapid developments of minimally-invasive surgery and visually assisted micro-robotics in surgery. New investigation fields are:

- in cardiovascular medicine: coronary angioscopy, cardiac valves surgery, angioscopy of peripheral vessels, atherosclerosis,

- in neurosurgery: anevrism or tumour microsurgery, lumbar microsurgery,

- in gastroenterology: pancreatic canal, cholecystectomy,

- in urology: lithotripsy without anaesthesia

- in gynaecology: diagnosis of endometriosis

- in paediatrics: microsurgery of neonates...

- in anaesthesia for the control of intubation,

- in Ophtalmologie: analysis of retinopathies, guidance

if microsurgical interventions,

- in arthroscopy for the surgery of small joints.

The question of the image quality evaluation and optimization in microendoscopy $(\varnothing<0.5 \mathrm{~mm})$ and of its improvements is a of prime importance in the concept of microendoscopy. An example of images obtained in a small artery of the foot is given in fig 2. Microendoscopy suffers from the limitations imposed by the physical laws governing the propagation of light in the fibers, in the lens and at the interfaces between the different optical components. The physics of light propagation through the microlens and the multicore fiber imposes an upper limit to the Modulation Transfer Function (MTF), and to the contrast which are, if the noise is neglected, the basic parameters involved in the image quality.

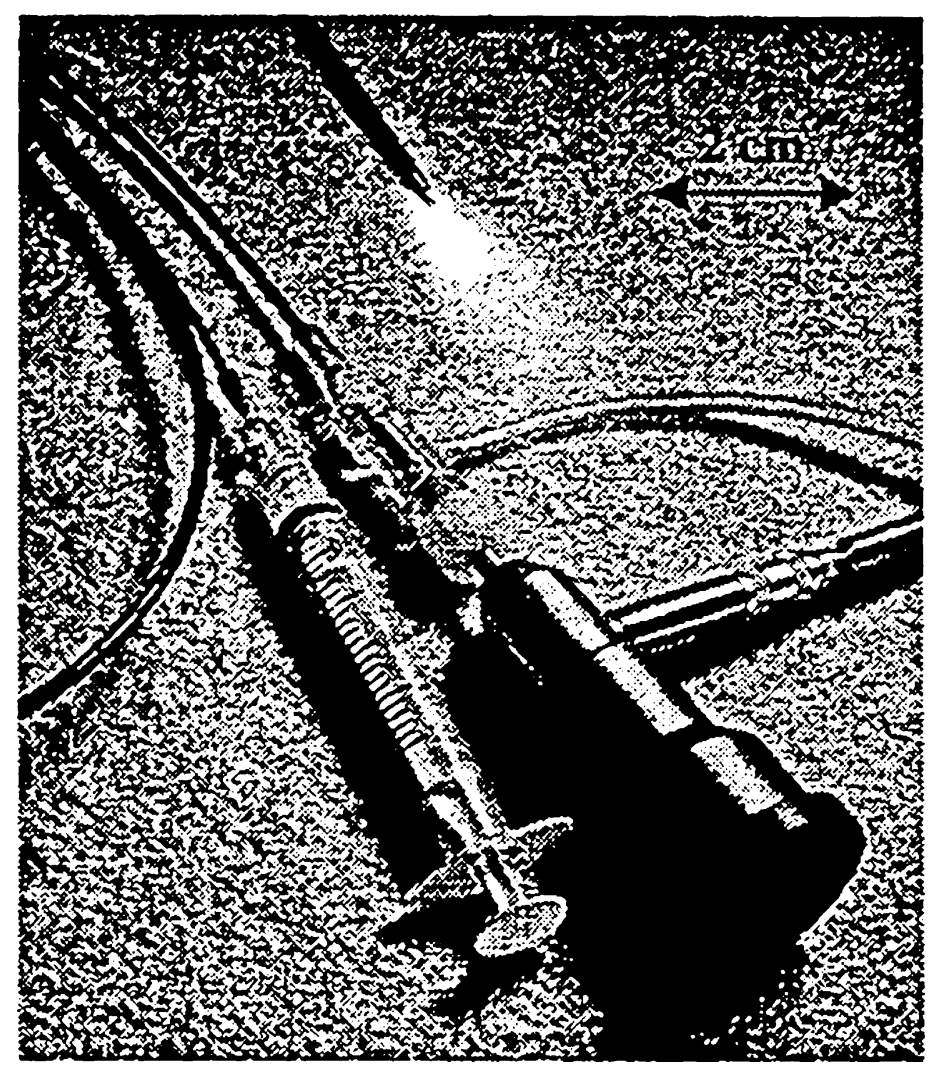

Fig. I Example of a sub-millimetre flexible endoscope

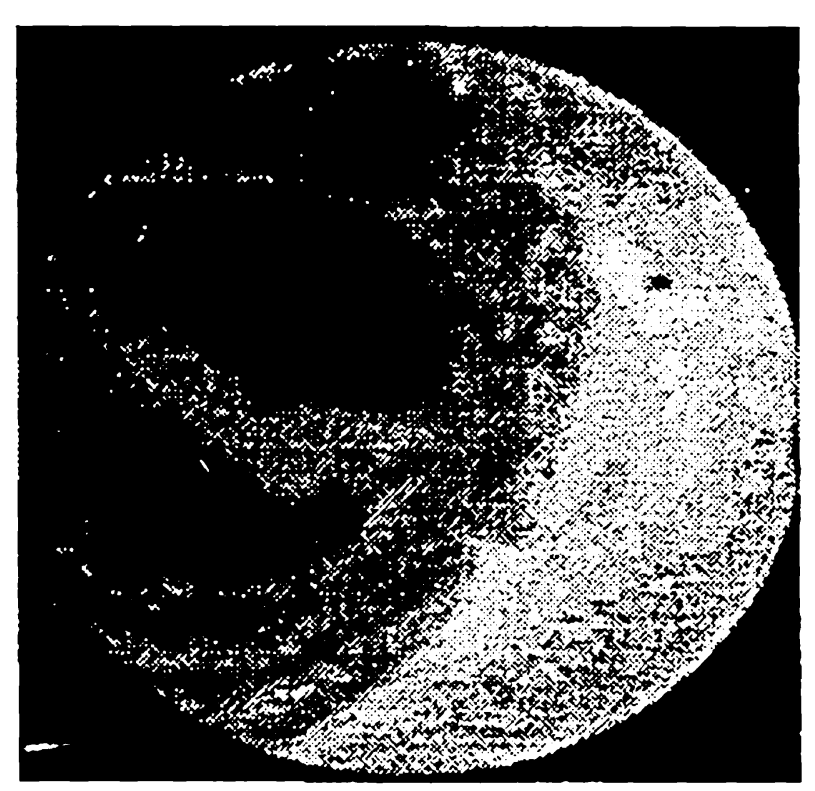

Fig. 2 Microendoscopic view of an arterial lumen, showing a dissection of the intima.

\section{METHOD}

An approach has been proposed to evaluate the MTF by measuring the transmitted contrast of Ronchi rulings [1][2][3] In the so-called "static scanning" method upper and lower analytical limits are derived with a good agreement with the measured results.

The MTF of the Selfoc Grin Lens has been measured with a set of Ronchi rulings, positioned at a controllable distance of the Selfoc Grin lens. A microscope objective focuses the transmitted image by the Selfoc Grin lens on a CCD camera. The intensity values of the captured image are vertically averaged in the central region of the 
GRIN lens. The contrast is calculated for the successive intensity extrema and the averaged values are assumed to represent the maximal MTF value. The magnification is calculated in order to have the spatial frequency of the Ronchi ruling images

\section{RESULTS:}

The MTF has been carefully evaluated by a precise and reproducible method and reveals significant differences between the various multicore fibers (see fig 3.) The differences of MTF's are due to the differences in the conception and realizations of the multicore fibers: intercore distances, diameters of the cores, numerical apertures [4]. All these factors influence the cross talk between the light guiding cores and influence therefore the fiber MTF.

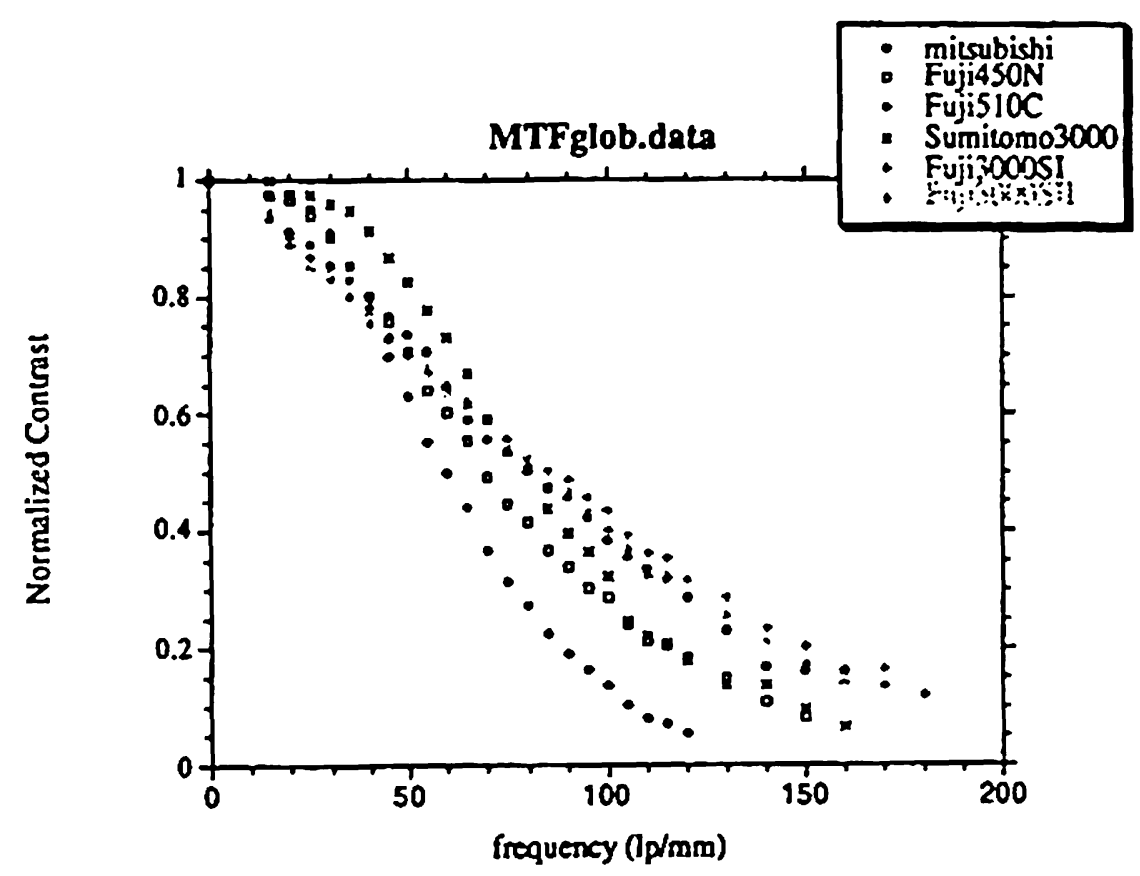

Fig.3: MTF's of various types of multicore fibers

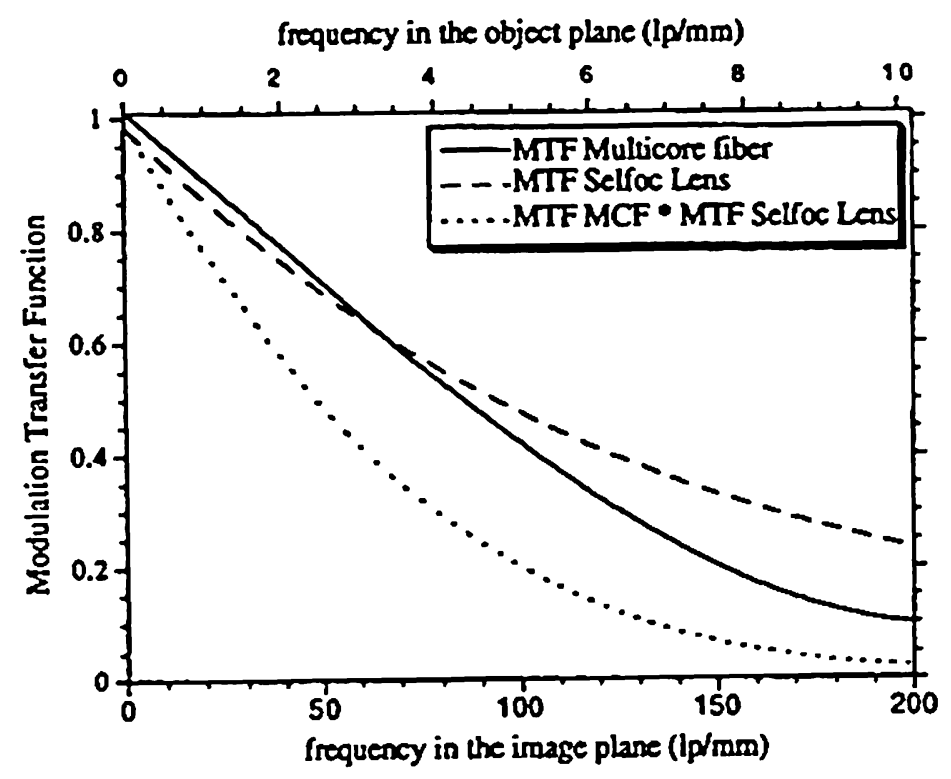

Fig.4: comparison between one of the best MTF's for the multicore fiber and the MTF of the selfoc lens.

The MTF of one of the most performant multicore fibers that we have tested can be compared with the MTF of the selfoc lens (see fig 4.). It must be observed that their values are pretty similar, proving that none of these two essential optical components of the microendoscope is responsible, alone, of the degradation of the image quality. The drop of the MTF of the multicore fiber is however more pronounced at high spatial frequencies, which is due to the limitations associated with the Nyquist law and the residu- al crosstalk between adjacent cores.

\section{DISCUSSION AND CONCLUSION:}

The optimization of the image quality in microendoscopy is the result of a trade-off between the different factors which play a significant role in the design and realization of the microendoscope: among them we must mention the geometrical and chromatic aberrations of the GRIN or Selfoc ${ }^{\mathrm{TM}}$ lens, the density of the cores and importance of the crosstalk in the multicore fiber [5], and some uncontrollable and misunderstood factors related to the interfaces, in particular the adhesives used to fix the GRIN lens. As $\mathrm{far}$ as the multicore fiber is concerned, a significant improvement of the image quality has been be obtained through adequate optical handling of the endoscopic image, such as the use of optical masks [6]. Another successful approach has been developed by processing electronically the image: image correction and restoration have proved useful to optimize the microendoscopic image. New assembly techniques of micro-optical components of the endoscope are also currently developed to improve the image quality.

\section{ACKNOWLEDGMENTS:}

This work is supported by the CTI, project "Développement d'un endoscope miniaturisé de nouvelle conception", in cooperation with Renaud and Nicolas Croisy (Andromis, Genève)

\section{REFERENCES:}

[1] Conde R., Depeursinge C., Coquoz O. and Taleblou F. (1993), SPIE Proceedings on Delivery Systems, 2084, 87-98.

[2] Conde R., Depeursinge C., Coquoz O. and Taleblou F., SPIE Proceedings on Speciality Fibers for Biomedical and Systems Applications, 2131, 215-223 (1994).

[3] Conde R., Depeursinge C., Coquoz O. and Taleblou F., SPIE Proceedings on Medical Applications of Lasers II, 2327, 356363 (1994).

[4] Conde R., Depeursinge C., Gisin B., Gisin N. and Groebli B., Pure and Applied Optics, 5, pp. 269-274, 1996.

[5] R. Conde, O. Coquoz, C. Depeursinge, F. Taleblou, R. Salathé, Optical Engineering, 34, 7, pp. 2092-2102, (1995).

[6] R.Conde, Thèse No 1395, EPFL, 1995. 